\title{
Clinical efficacy of various anti-hypertensive regimens in hypertensive women of Punjab; a longitudinal cohort study
}

\author{
Muhammad Umair ${ }^{1}$, Mobasher Ahmad ${ }^{1}$, Hamid Saeed ${ }^{1,2^{*}}$ (D) Zikria Saleem $^{2,3}$ and Fatima Tauqeer ${ }^{4}$
}

\begin{abstract}
Background: Gender wise differences exist in anti-hypertensive treatment outcomes, yet still un-explored in Pakistan. Thus, we aimed to estimate the clinical efficacy of four different anti-hypertensive regimens in hypertensive women of Punjab, Pakistan.
\end{abstract}

Methods: A longitudinal cohort study of 12 months duration was conducted by enrolling 300 hypertensive women on four anti-hypertensive regimens. Chi-square for significance, logistic regression for association and multilevel regression for changes in outcomes were used.

Results: Majority of subjects were $<60$ years of age, weighing $>65 \mathrm{Kg}$, having family history, married and hailing from urban areas, with diabetes as the most common comorbidity. Hypertension, adjusted for covariates, was significantly associated with salt intake (OR:2.27, $p<0.01)$ and physical activity $(\mathrm{OR} ; 2.16, p<0.01)$. High-risk subjects, compared to low-risk, were consuming more fat (OR;1.54), meat (OR; 2), salt (OR; 2.48$)$ and even vegetables/fruits $(\mathrm{OR} ; 3.43)$. Compared to baseline, the maximum reduction in BP was observed with combination therapy, $\mathrm{N}$ GITS+LTN + HCT (SBP; $-50.17, p<0.01$, DBP; $-16.55, p<0.01$ ), followed by N-GITS alone (SBP; $-28.89, p<0.01$, DBP; $-12.21, p<0.01$ ). Compared to baseline, adjusted for treatment effects, significant reductions in SBP (low-risk; - 17.92, $p<0.01$ high-risk; - 19.48, $p<0.01$ ) and DBP (low-risk; - 17.92, $p<0.01$, high-risk; - 19.48, $p<0.01$ ) were observed in low and high risk patients. Among all four cohorts, orthostatic hypotension and edema were common in N-GITS+LTN + HCT only, but variable effects were observed on biochemical values; urea, BSR and creatinine.

Conclusion: In conclusion, compared to a single agent, combination therapy conferred improved BP controls followed by N-GITS alone in low and high risk women with manageable side effects.

Keywords: Hypertension, Women, Punjab, Pakistan, Nifedipine-GITS, Losartan, Combination therapy

\section{Background}

Hypertension is a public health issue and a major cause of morbidity and mortality. It is responsible for almost $13 \%$ of all deaths and $3.7 \%$ total disability adjusted life years [1]. It is estimated that of all the deaths (17

\footnotetext{
* Correspondence: hamid.pharmacy@pu.edu.pk

'Section of Pharmacology, University College of Pharmacy, University of the Punjab, Allama Iqbal Campus, Lahore 54000, Pakistan

${ }^{2}$ Section of Pharmaceutics, University of the Punjab, Allama Iqbal Campus, Lahore 54000, Pakistan

Full list of author information is available at the end of the article
}

million) globally due to cardiovascular diseases, 50\% ( 9.4 million) are due to complications related to hypertension [2]. It is now well documented that gender base differences exist in the pathophysiology of hypertension, probably due to age related differences in arterial tree between the sexes [3]. However, to date, the data is scarce that may demonstrate gender wise differences in blood pressure responses towards anti-hypertensive agents [4].

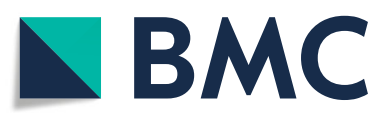

(c) The Author(s). 2020 Open Access This article is licensed under a Creative Commons Attribution 4.0 International License, which permits use, sharing, adaptation, distribution and reproduction in any medium or format, as long as you give appropriate credit to the original author(s) and the source, provide a link to the Creative Commons licence, and indicate if changes were made. The images or other third party material in this article are included in the article's Creative Commons licence, unless indicated otherwise in a credit line to the material. If material is not included in the article's Creative Commons licence and your intended use is not permitted by statutory regulation or exceeds the permitted use, you will need to obtain permission directly from the copyright holder. To view a copy of this licence, visit http://creativecommons.org/licenses/by/4.0/ The Creative Commons Public Domain Dedication waiver (http://creativecommons.org/publicdomain/zero/1.0/) applies to the data made available in this article, unless otherwise stated in a credit line to the data. 
Literature regarding blood pressure control in both men and women are contradictory, a few studies suggest that women are more likely to be treated but less likely to achieve blood pressure control [5, 6]. However, age dependent relationship between blood pressure control and gender has been documented - poor blood pressure control in younger men and older women [7]. Numerous studies have shown that the risk of heart failure and mortality rate due to hypertension is greater in women compared to men $[8,9]$. Contrary to standard guidelines, numerous observational studies have shown that both men and women are treated with different antihypertensive agents - women with diuretics or beta blockers and men with ACE inhibitors or calcium channel blockers $[5,6]$. Women above 50 years of age exhibited greater protection from stroke on calcium channel blockers rather than using ACEIs [10]. According to European guidelines, calcium channel blockers are considered the only class of antihypertensive agents that can produce desirable effects in combination with other four classes of antihypertensive drugs [11].

Among others, Nifedipine gastrointestinal therapeutic system (N-GITS) provides sustained $24 \mathrm{~h}$ antihypertensive effect with no overt cardio-acceleration
[12]. The efficacy of Nifedipine GITS has been established in numerous studies, alone or in combination, in hypertension and angina - supported by the outcomes of INSIGHT and ACTION trials [13, 14]. Results from a randomized control trial, ADVISE study, clearly demonstrated that blood pressure controls were better in Asian population, both males and females, on nifedipine GITS plus valsartan compared to higher doses of valsartan alone, even when stratified for smoking and systolic blood pressure (SBP) [15].

Despite higher prevalence of hypertension among women of Pakistan with associated risk of cardiovascular disease [16, 17], scanty of literature evidences exist regarding the clinical efficacy and safety of various antihypertensive agents in hypertensive women - not a single from Pakistan and almost negligible from South Asian region. Thus, the aim of the present study was to evaluate the clinical efficacy and safety of four antihypertensive regimens, namely; losartan (LTN), N-GITS (nifedipine-GITS), LTN + hydrochlorothiazide (LTN + $\mathrm{HCT}$ ) and LTN + HCT + N-GITS, in low and high risk hypertensive women of Punjab, Pakistan.

\section{Methods}

The layout of cohort study design is described in Fig. 1.

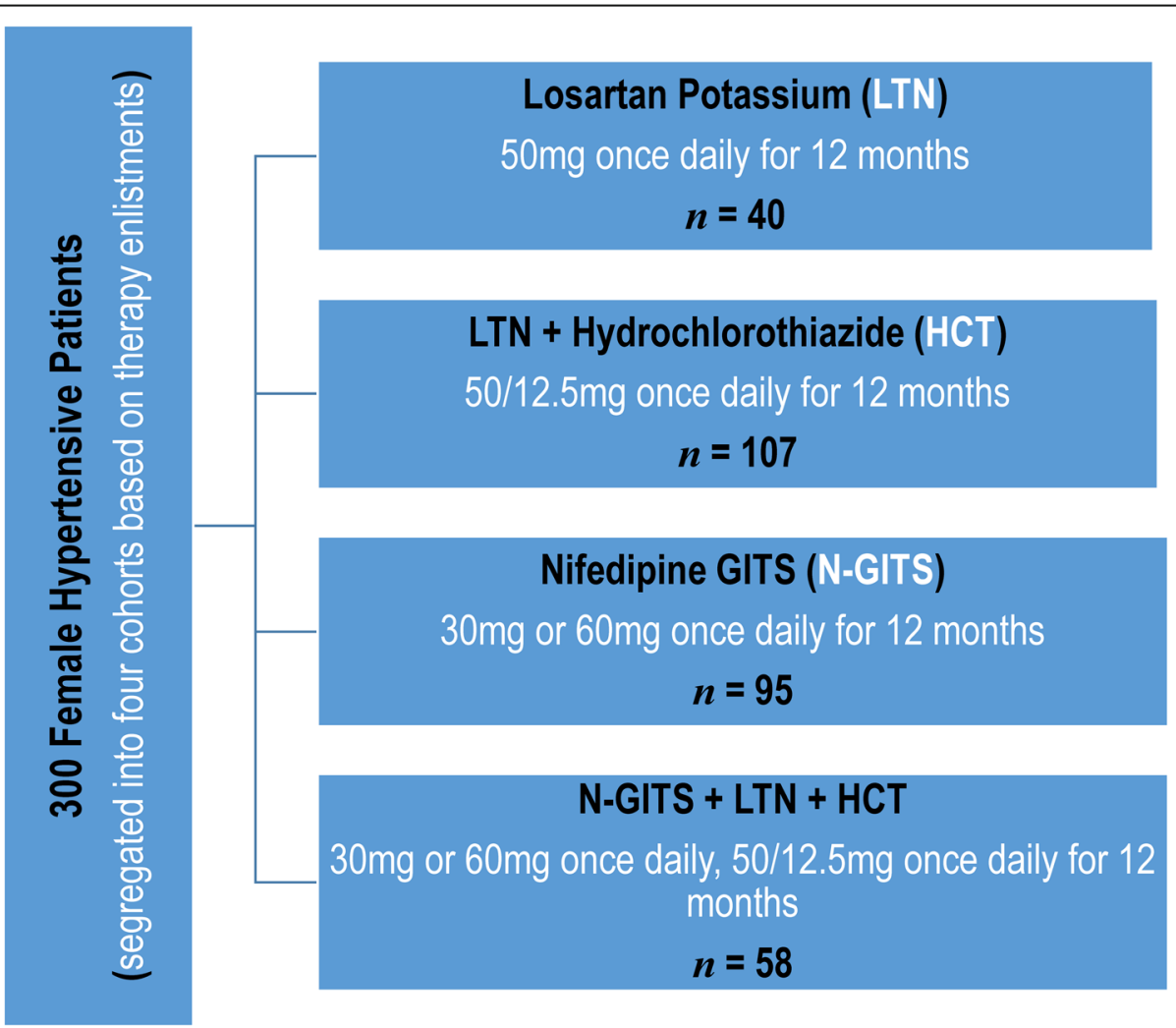

Fig. 1 A Brief Layout of the Study Design 


\section{Study design}

A longitudinal cohort study was conducted by enrolling 300 hypertensive women from Fauji foundation hospital, Lahore. The study period was 1 year, i-e., June 2016May 2017. Hypertension was documented as per clinician's report $-150 / 90 \mathrm{mmHg}$ or higher for patients 60 years or above without any comorbidity and 140/90 $\mathrm{mmHg}$ or higher for adults below 60 years of age as per JNC8 guidelines [18].

\section{Sample size}

Sample size was calculated based on disease prevalence (hypertension) [19] in Pakistani women, which was around 39\%, as of June 2017 [20]. The sample size was found to be 363 using 95\% confidence interval and 5\% precision. However, we didn't get more than 300 patients due specific therapy enlistment and selection of a single health facility (Fauji foundation hospital).

\section{Study cohorts}

As per study objectives, the study cohorts, i-e., Losartan (LTN) group $(n=40)$, Nifedipine-GITS $(\mathrm{N}-\mathrm{GITS})$ group $(n=95), \mathrm{LTN}+$ hydrochlorothiazide $(\mathrm{HCT})$ group $(n=$ $107)$ and LTN + N-GITS + HCT group $(n=58)$, were identified from Hospital Information System (HIS) in consultation with the medical practitioner providing treatments to the patients reporting to the hospital for the year, 2016. Most of the patients in four cohorts were already on enlisted therapies before their enrollment in the study. After enrollments following study inclusion and exclusion criteria, the baseline clinical and laboratory parameters were recorded from patient's medical files extracted via hospital information system.

\section{Follow ups}

After documenting baseline parameters, these 300 hypertensive women, started on four anti-hypertensive regimens mentioned above, were observed from June 2016 till May 2017 with total of 3 follow ups - each follow-up after every 3 months. All the parameters recorded at baseline were documented at each follow up to examine therapy effects.

\section{Risk assessment}

Subjects having co-morbid conditions were considered high risk, while hypertension alone cases were considered low risk.

\section{Study settings}

The Fauji foundation hospital, established in 2001 and governed by Pakistani army, was selected because it's one of the leading hospitals of Pakistan that receive new and referral hypertensive patients from all over the province, Punjab, with complete documentation of patient's medical records [21]. It's a 250 bedded hospital spread over an area of 6.5 acer in cantonment, Lahore, Punjab, Pakistan. Hospital provides free medical services and medication to its past and present employees - exmilitary service men.

\section{Participants}

A total of 300 subjects were registered in the study from Fauji Foundation Hospital, Lahore, Pakistan. The subjects were enrolled as per study inclusion and exclusion criteria.

\section{Inclusion criteria}

All hypertensive women above 18 years of age, with not more than two co-morbid conditions, irrespective of ethnicity, area of residence, social status and on specified therapy protocols as mentioned in study design were included in the study.

\section{Exclusion criteria}

All hypertensive women having mental health issues affecting cognition, more than two co-morbid conditions and not willing to participate in the study were excluded from the study. No exclusion was made based on patient's altered lab values.

\section{Variables \\ Treatments}

Out of 300 hypertensive women, 40 were on Losartan potassium (LTN) $50 \mathrm{mg}$ daily, 107 were on LTN + hydrochlorothiazide (HCT) 50/12.5 mg daily, 95 were on Nifedipine GITS 30 or $60 \mathrm{mg}$ daily and 58 were on Nifedipine GITS + LTN + HCT 30 or $60 \mathrm{mg}+50 / 12.5 \mathrm{mg}$ daily, all for the period of 12 months (Fig. 1).

\section{Outcome measures}

The primary efficacy endpoints were mean changes in systolic (SBP) and diastolic blood pressure (DBP) from the baseline values in each arm measured at each follow up till final follow up. BP was measured using manual $\mathrm{BP}$ measuring devices.

\section{Safety endpoints}

Safety parameters were estimated based on the reported frequencies of treatment related adverse reactions (ADRs). All adverse effects that occurred throughout the study period were recorded and evaluated for their seriousness and relation to the drugs. Notable ADRs, documented based on their associations with treatment protocols, include, dry cough, headache, orthostatic hypotension and edema. 


\section{Life style measures}

Physical activity A daily physical activity of 30 min was considered normal, while a daily activity of less than 30 min was considered as low.

Food consumption Food consumption was documented to estimate their possible association with hypertension under the following sub-headings.;

- Red or white meat: consumption of red or white meat was scored as 1-2 times and $>2$ times in a day - at least $35-70 \mathrm{~g}$ was considered 1-2 times per day, while more than $70 \mathrm{~g}$ was considered $>2$ times a day.

- Vegetables and fruits: it was recorded as 1-2 times and 2-3 times a day - at least $1 / 3$ rd portion of the meal or 1-1.5 cup of vegetables \& fruits, fresh or cooked was considered as 1-2 time a day, while more than twice was considered $>2$ times a day.

- Salt Intake: Salt intake was documented as normal if daily intake was equal to 1 teaspoon. i-e., $6 \mathrm{~g}$, while salt intake of $\leq 4.5 \mathrm{~g}$ was considered low.

- Fat Intake: consumption of trans and saturated food, snacks, fast food, creamers and cakes, was considered high fat consumption, while avoiding these food items was documented taken as low fat consumption [22]..

\section{Data collection}

Data collection form was designed fulfilling all the necessary objectives of the study. Utilizing patient's medical files, patient's baseline demographics (name, age, gender, weight, address, BMI, occupation, marital status, and number of children), lifestyle patterns (physical activity and food consumption), clinical variables (systolic blood pressure (SBP) and diastolic blood pressure (DBP) in $\mathrm{mmHg}$, history of cardiovascular events, the presence of comorbidities and laboratory biochemical values (blood sedimentation rate (BSR), urea and creatinine) were documented. Moreover, history of illness, disease symptoms and possible drug related side effects were also recorded.

On every follow up, after every 3 months, again pertinent clinical, SBP and DBP, and laboratory biochemical data, i-e,. BSR, urea, creatinine and safety endpoints were documented under the supervision of a medical practitioner.

\section{Data analysis}

The socio-demographic characteristics of the patients segregated to four different anti-hypertensive therapies were analyzed and compared using StataSE14 and SPSS (IBM, version 21). Descriptive statistics were performed to estimate the frequencies of all socio-demographic variables and food consumption using cross-tabulation. A linear mixed effect model was used to evaluate the changes over time in SBP, DBP, urea, serum creatinine, $\mathrm{Hb}$ and random blood glucose levels.

Data for the primary outcomes, collected at 5 different study time points, from baseline to final follow up, were assumed to be clustered within patients. It is therefore unreasonable to assume that these data were independent. To account for the clustering effect of these data, we fitted linear multilevel models. Thus, a two-level model with random intercept and random effect of time on patients at level 2 was considered. The models were used to assess the mean changes of the primary outcomes at each study time point relative to baseline. To understand the factors that were associated with being in the low or high risk group, binary logistic regression models within the generalized linear regression model (GLM) were fitted to the data. All models were fitted using StataSE 14. An alpha value of o.0.5 or less was considered statistically significant.

\section{Results}

\section{Patient's demographics}

Patient's basic characteristics are summarized in Table 1. Data suggested that frequency distribution was significantly different among all the treatment protocols with regards to age, mostly < 60 years of age (LTN; $85 \%, \mathrm{~N}$ GITS; 49.5\%, LTN + HCT; 71\%, N-GITS+LTN + HCT; $51.7 \%, p<0.01$ ), family history; mostly had no familial link (LTN; 60\%, N-GITS; 76.8\%, LTN + HCT; 57\%, NGITS+LTN + HCT; 56.9\%, $p=0.01$ ), education; mostly having secondary education $(p<0.01)$ and physical activity $(p<0.01)$; more than $44 \%$ of the subjects in each treatment arm claimed to have normal physical activity (Table 1). Similarly, majority of the patients on protocols other than LTN were consuming red or white meat 1-2 times a day (N-GITS; 58.9\%, LTN + HCT; 80.4\%, NGITS+LTN + HCT; 91.4\%, $p<0.01)$ and had low salt intake (N-GITS; 53.7\%, LTN + HCT; 73.8\%, N-GITS+ LTN + HCT; $86.2 \%, p<0.01)$ (Table 1).

\section{Treatment outcomes and laboratory biochemical values; baseline vs follow ups}

Data on treatment outcomes and laboratory biochemical values, baseline (BL) vs follow up, are summarized in Table S1. Data revealed that mean DBP and hemoglobin baseline values were not different among all four cohorts. However, considerable differences existed among cohorts in the mean baseline values of SBP (LTN; 141.87, N-GITS; 156.52, LTN + HCT; 153.73, LTN + NGITS+HCT; $184.05 \mathrm{mmHg}$ ), serum creatinine, serum urea and blood glucose levels (Table S1). When it comes to treatment effects, in last (12 months) follow up, all 
Table 1 Patient's Basic Demographics and Life Style Patterns

\begin{tabular}{|c|c|c|c|c|c|}
\hline \multirow[t]{2}{*}{ Characteristics } & \multicolumn{4}{|c|}{ Anti-hypertensive Protocols } & \multirow[t]{2}{*}{$p$-values } \\
\hline & $\operatorname{LTN} n=40(\%)$ & N-GITS $n=95(\%)$ & LTN + HCT $n=107(\%)$ & $\mathrm{N}$-GITS + LTN + HCT $n=58(\%)$ & \\
\hline \multicolumn{6}{|l|}{ Age (years) } \\
\hline$<60$ & $34(85.0)$ & $47(49.5)$ & $76(71.0)$ & $30(51.7)$ & \multirow[t]{2}{*}{$<0.01^{* *}$} \\
\hline$\geq 60$ & $6(15.0)$ & $48(50.5)$ & $31(29.0)$ & $28(48.3)$ & \\
\hline \multicolumn{6}{|l|}{ Body Weight (kg) } \\
\hline$\leq 65$ & $19(47.5)$ & $40(42.1)$ & $58(54.2)$ & $22(37.9)$ & \multirow[t]{2}{*}{0.17} \\
\hline$>65$ & $21(52.5)$ & $55(57.9)$ & $49(45.8)$ & $36(62.1)$ & \\
\hline \multicolumn{6}{|l|}{ Marital Status } \\
\hline Married & $39(97.5)$ & $89(93.7)$ & $100(93.5)$ & $54(93.1)$ & \multirow[t]{2}{*}{0.80} \\
\hline Single & $1(2.5)$ & $6(6.3)$ & $7(6.5)$ & $4(6.9)$ & \\
\hline \multicolumn{6}{|l|}{ Area of Residence } \\
\hline Urban & $27(67.5)$ & $60(63.2)$ & $66(61.7)$ & $37(63.8)$ & \multirow[t]{2}{*}{0.93} \\
\hline Rural & $13(32.5)$ & $35(36.8)$ & $41(38.3)$ & $21(36.2)$ & \\
\hline \multicolumn{6}{|l|}{ Number of Children } \\
\hline$<3$ & $19(47.5)$ & $23(24.2)$ & $34(31.8)$ & 19 (32.8) & \multirow[t]{2}{*}{0.07} \\
\hline$\geq 3$ & $21(52.5)$ & $72(75.8)$ & $73(68.2)$ & $39(67.2)$ & \\
\hline \multicolumn{6}{|l|}{ Family History } \\
\hline Yes & $16(40.0)$ & $22(23.2)$ & $46(43.0)$ & $25(43.1)$ & \multirow[t]{2}{*}{$0.01^{*}$} \\
\hline No & $24(60.0)$ & $73(76.8)$ & $61(57.0)$ & $33(56.9)$ & \\
\hline \multicolumn{6}{|l|}{ Occupation } \\
\hline Housewife & $32(80.0)$ & $93(97.9)$ & $99(92.5)$ & $56(96.6)$ & \multirow[t]{2}{*}{$<0.01^{* *}$} \\
\hline Employed & $8(20.0)$ & $2(2.1)$ & $8(7.5)$ & $2(3.4)$ & \\
\hline \multicolumn{6}{|l|}{ Education } \\
\hline Secondary & $13(32.5)$ & $71(74.7)$ & $66(61.7)$ & $40(69.0)$ & \multirow[t]{2}{*}{$<0.01^{* *}$} \\
\hline Above Secondary & $27(67.5)$ & $24(25.3)$ & 41 (38.3) & $18(31.0)$ & \\
\hline \multicolumn{6}{|l|}{ Food Consumption } \\
\hline \multicolumn{6}{|l|}{ Red or White meat } \\
\hline $1-2$ times & $17(42.5)$ & $56(58.9)$ & $86(80.4)$ & $53(91.4)$ & \multirow[t]{2}{*}{$<0.01^{* *}$} \\
\hline$>2$ times & $23(57.5)$ & $39(41.1)$ & 21 (19.6) & $5(8.6)$ & \\
\hline \multicolumn{6}{|c|}{ Vegetables and Fruits } \\
\hline $1-2$ times & $8(20.0)$ & $12(12.6)$ & $9(8.4)$ & $6(10.3)$ & \multirow[t]{2}{*}{0.26} \\
\hline$>2$ times & $32(80.0)$ & $83(87.4)$ & 98 (91.6) & $52(89.7)$ & \\
\hline \multicolumn{6}{|l|}{ Fat Intake } \\
\hline Low & $25(62.5)$ & $76(80.0)$ & $90(84.1)$ & $56(96.6)$ & $<0.01^{* *}$ \\
\hline Normal & $15(37.5)$ & $19(20.0)$ & $17(15.9$ & $2(3.4)$ & \\
\hline Salt Intake & & & & & \\
\hline Low & $12(30.0)$ & $51(53.7)$ & $79(73.8)$ & $50(86.2)$ & $<0.01^{* *}$ \\
\hline Normal & $28(70.0)$ & $44(46.3)$ & $28(26.2)$ & $8(13.8)$ & \\
\hline Physical Activity & & & & & \\
\hline Low & $9(22.5)$ & 49 (51.6) & $35(32.7)$ & $32(55.2)$ & $<0.01^{* *}$ \\
\hline Normal & $31(77.5)$ & $46(48.4)$ & $72(67.3)$ & $26(44.8)$ & \\
\hline
\end{tabular}


four therapeutic regimens had significant impact on SBP (LTN: BL; 141.87, 12-months; 123.25, $p=0.001, \mathrm{~N}$ GITS: BL; 156.52, 12-months; $127.63, p=0.001, \mathrm{LTN}+$ HCT: BL; 153.73, 12-months; 128.54, $p=0.001, \mathrm{LTN}+$ N-GITS + HCT: BL; 184.05, 12-months; 133.88, $p=$ 0.001 ) and DBP (LTN: BL; 90.12, 12- months; 80.83, $p=$ 0.001, N-GITS: BL; 93.51, 12-months; 81.28, $p=0.001$, LTN + HCT: BL; 92.22, 12-months; 82.12, $p=0.001$, LTN + N-GITS + HCT: BL; 100.44， 12-months; 84.04, $p=0.001)$. As for laboratory biochemical values, compared to baseline vs 12-months, mean serum creatinine levels were increased in LTN group only and mean serum urea levels were increased in N-GITS (31.27, $p=$ $0.01), \quad \mathrm{LTN}+\mathrm{HCT} \quad(30.85, \quad p=0.001)$ and LTN + NGITS+HCT (36.03, $p=0.001)$ groups. No significant effects of all four regimens were observed on $\mathrm{Hb}$ levels (Table S1).

\section{Frequency of co-morbid conditions and therapy related side effects}

As shown in Figure S1, diabetes was the most frequent single co-morbid condition (13.6\%) followed by anemia (11\%), angina (7.7\%) and ischemic heart disease $(3 \%)$. In more than one co-morbid category, diabetes + angina (2.7\%) and diabetes + anemia (1.3\%) were the notable co-morbid conditions (Fig. S1). As for the side effects, in combination therapy; N-GITS+ LTN + HCT, orthostatic hypertension (15.8\%) was most frequently observed side effect followed by edema (6.9\%) (Fig. S2). In LTN + HCT group, orthostatic hypertension (8.4\%) and headache $(8.4 \%)$ were reported with similar frequencies. While in monotherapy, patients on N-GITS experienced edema (5.2\%), headache $(4.7 \%)$ and orthostatic hypertension (3.2\%), yet with lower frequencies compared to combination therapy (Fig. S2).

\section{Association of lifestyle patterns with overall hypertensive and high risk population}

As shown in Table 2, hypertension was significantly associated with red/white meat intake $>2$ times a day (OR; $2, p=0.01)$, weight $\geq 65 \mathrm{Kg}(\mathrm{OR} ; 1.63, p=0.04)$, vegetables and fruits intake $>2$ times a day (OR; 3.34, $p=$ $0.001)$, salt intake (OR; $2.48, p<0.01)$ and physical activity (OR; $2.79, p=0.001)$. When adjusted for covariates, only salt intake, vegetables and fruit intake, and physical activity demonstrated significant associations with hypertension (Table 2).

When patients were segregated into low and high risk groups, as described in method section, forest plot revealed that high risk women were more likely to have normal salt intake (OR; 2.48, ref; low intake), meat intake > 2 times (OR; 2.02, ref; $1-2$ times), vegetables and fruits intake $>2$ times (OR; 3.43, ref; $1-2$ times) and
Table 2 Association of Lifestyle Patterns in Women with Hypertension

\begin{tabular}{|c|c|c|c|c|}
\hline \multirow{2}{*}{ Factors } & \multicolumn{2}{|l|}{ Bivariate analysis } & \multicolumn{2}{|l|}{ Adjusted } \\
\hline & OR $(95 \% \mathrm{Cl})$ & $\boldsymbol{p}$-value & OR $(95 \% \mathrm{Cl})$ & $\boldsymbol{p}$-value \\
\hline \multicolumn{5}{|c|}{ Salt intake/day (ref: Low) } \\
\hline Normal & $2.48(1.50,4.11)$ & $<0.01^{* *}$ & $2.27(1.20,4.31)$ & $0.01^{*}$ \\
\hline \multicolumn{5}{|c|}{ Red/White meat intake/day (ref: $1-2$ times) } \\
\hline$>2$ times & $2(1.19,3.42)$ & $0.01^{*}$ & $1.81(1.1,3.81)$ & 0.57 \\
\hline \multicolumn{5}{|c|}{ Vegetables and fruits intake/day (ref: $1-2$ times) } \\
\hline$>2$ times & $3.34(1.61,7.3)$ & $<0.01^{* *}$ & $3.27(1.6,6.71)$ & $<0.01^{* *}$ \\
\hline \multicolumn{5}{|c|}{ Fat intake/day (ref: Low) } \\
\hline Normal & $1.54(0.82,2.86)$ & 0.18 & $1.43(0.77,2.91)$ & 0.75 \\
\hline \multicolumn{5}{|c|}{ Weight $(\mathrm{kg})($ ref: < 65 kg) } \\
\hline$\geq 65 \mathrm{~kg}$ & $1.63(1.03,2.60)$ & $0.04^{*}$ & $1.56(0.93,2.62)$ & 0.10 \\
\hline \multicolumn{5}{|c|}{ Physical activity (ref: Low) } \\
\hline Normal & $2.79(1.74,4.50)$ & $<0.01^{* *}$ & $2.16(1.29,3.62)$ & $<0.01^{* *}$ \\
\hline
\end{tabular}

body weight $\geq 65 \mathrm{Kg}(\mathrm{OR} ; 1.54, \mathrm{ref} ;<65 \mathrm{Kg})$ compared to low risk subjects, despite normal physical activity and fat intake (OR; 1.54, ref; low intake) (Fig. 2).

\section{Changes in outcome measures at each follow up in hypertensive women}

To examine the changes in outcome measures at each follow up in comparison to baseline values, we fitted models with second-order interaction between therapy and time in order to investigate the changes of the primary outcomes over time (Table 3). The mean changes reported in Table 3 are within various anti-hypertensive therapies relative to baseline, adjusted for socio-demographic factors. All treatment protocols, LTN, N-GITS, LTN + HCT and N-GITS + LTN + HCT demonstrated significant reduction in SBP starting at 3 months of follow up till 12 months, though maximum reduction was observed in N-GITS $+\mathrm{LTN}+\mathrm{HCT}$ therapy group $(\beta ;-50.17, p<0.001)$ followed by N-GITS $(\beta ;-28.89, p<0.001)$, a similar trend was observed for DBP - N-GITS + LTN + HCT therapy group $(\beta ;-16.55, p<0.001)$ followed by $\mathrm{N}$ GITS $(\beta ;-12.21, p<0.001)$ (Table 3$)$. Compared to baseline laboratory biochemical values, at final follow up, 12 months, BSR levels exhibited maximum reduction in N-GITS $(\beta ;-25.29, p<0.001)$ and N-GITS + LTN + HCT therapy $(\beta ;-24.93, p<0.001)$ groups, while urea levels were increased in N-GITS + LTN + $\operatorname{HCT}(\beta ; 3.47, p<0.001)$ and LTN + HCT $(\beta ; 5.16$, $p<0.001$ ) groups (Table 3 ). Interestingly, only $\mathrm{N}$ GITS treatment resulted in modest changes in blood urea and creatinine levels, which started to appear in 3rd follow up (Table 3). 


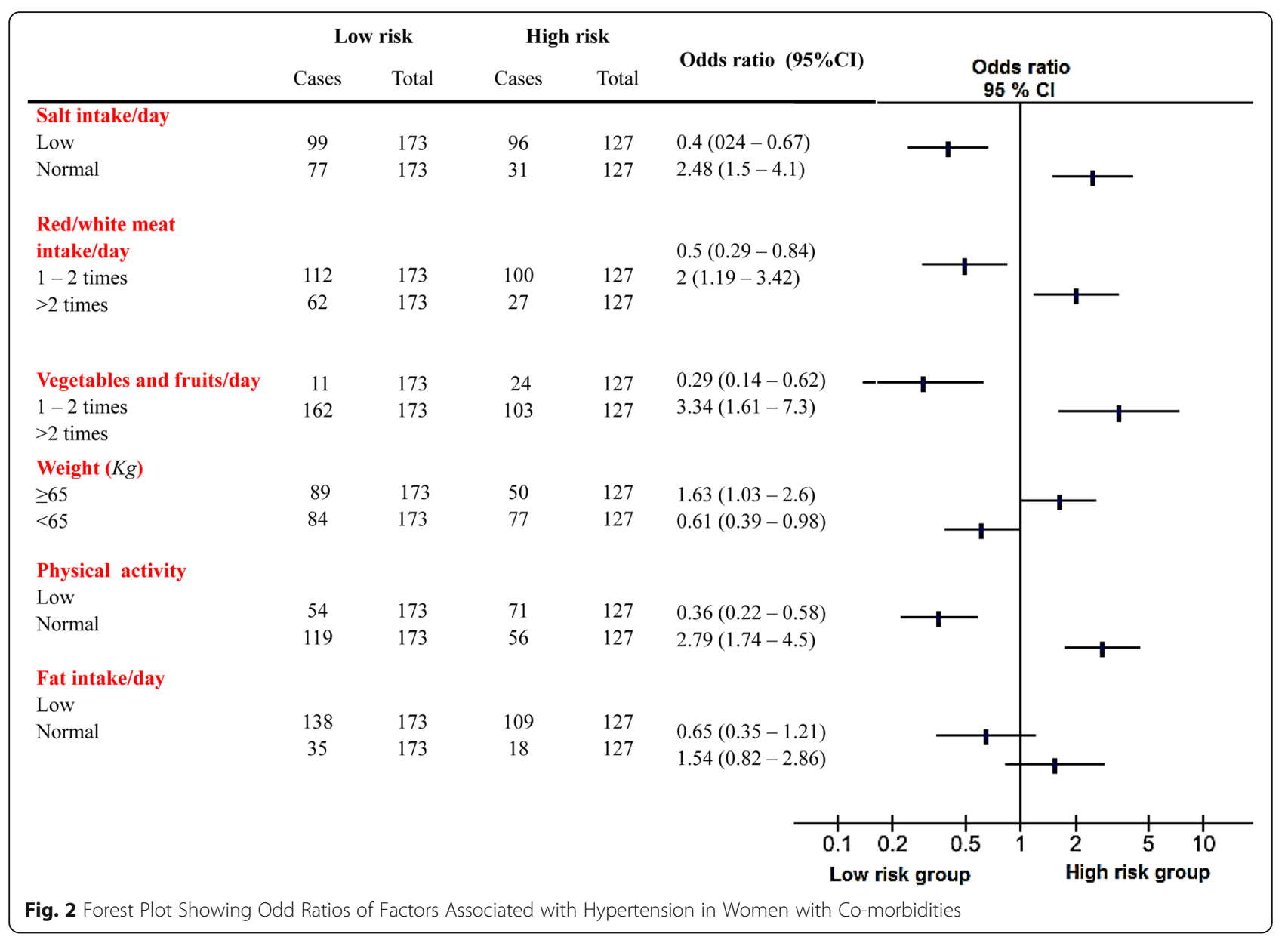

\section{Changes in outcome measures, low vs high risk hypertensive women}

Table 4 showed the changes in outcome measures, i-e., SBP, DBP, urea, BSR and creatinine within and between low (LR) and high risk (HR) hypertensive women, adjusted for treatment effects, at each follow up. In low and high risk groups, compared to baseline, we observed significant reduction in SBP (at 12 months: LR; $\beta$; 17.92, $p<0.001$, HR; $-19.48, p<0.001$ ) and DBP (at 12 months: LR; $\beta$; $-9.49, \quad p<0.001, \mathrm{HR} ;-10.12$, $p<0.001)$ at each follow up, which became maximum at final follow up (12 months). In both low and high risk groups, compared to baseline, changes in urea and creatinine were observed after 2nd and 3rd follow ups (Table 4). But regarding changes between the groups, low vs high risk, only $\operatorname{SBP}(\beta ;-1.55, p=0.03)$ demonstrated a significant change at final follow up (Table 4).

\section{Discussion}

Hypertension is a major risk factor of cardiovascular diseases (CVDs) and a major contributor to CVDs related deaths, approximately one death per minute among women in the United States [23]. According to recent estimates from Punjab, Pakistan, the prevalence of hypertension in women is $41 \%$, almost $10 \%$ higher than men [20]. However, not a single study from Pakistan has been reported to estimate the clinical efficacy of different anti-hypertensive agents in women of Punjab, Pakistan. In the present study it was observed that among hypertensive women of Punjab, Pakistan, diabetes is the most common co-morbid condition followed by anemia, angina and ischemic heart disease. Life style and dietary patterns demonstrated significant associations with high risk hypertensive subjects, such as consumption of salt, fat, red/white meat, vegetables and fruits, and physical activity in comparison to low risk hypertensive subjects. Among the four treatment cohorts, combination regimen; N-GITS + LTN + HCT, and single agent N-GITS demonstrated improved anti-hypertensive effects, on both SBP and DBP, however, the treatment related side effects, orthostatic hypotension and edema, were less frequently observed with monotherapy compared to combination therapy. Only N-GITS exhibited minimal effects on serum urea and creatinine levels.

As reported previously, with advance aging, in postmenopausal women above 65 years of age, the 
Table 3 Therapy Related Changes in Outcome Measures at Each Follow up Compared to Baseline Using Linear Multilevel Regression Model

\begin{tabular}{|c|c|c|c|c|c|c|c|c|}
\hline \multirow{2}{*}{$\begin{array}{l}\text { Changes in } \\
\text { Outcome Measures }\end{array}$} & \multirow{2}{*}{$\begin{array}{l}3 \text { Months } \\
\beta(95 \% \mathrm{Cl})\end{array}$} & \multicolumn{3}{|c|}{6 Months } & \multicolumn{2}{|l|}{9 Months } & \multicolumn{2}{|l|}{12 Months } \\
\hline & & $p$-value & $\beta(95 \% \mathrm{Cl})$ & $\boldsymbol{p}$-value & $\beta(95 \% \mathrm{Cl})$ & $\boldsymbol{p}$-value & $\beta(95 \% \mathrm{Cl})$ & $\boldsymbol{p}$-value \\
\hline \multicolumn{9}{|c|}{ Changes in SBP from baseline } \\
\hline LTN & $\begin{array}{l}-17.88(-20.25,- \\
15.50)\end{array}$ & $\begin{array}{l}< \\
0.01 * *\end{array}$ & $\begin{array}{l}-17.88(-20.25,- \\
15.50)\end{array}$ & $\begin{array}{l}< \\
0.01^{* *}\end{array}$ & $\begin{array}{l}-16.38(-18.75 \\
-14.00)\end{array}$ & $\begin{array}{l}< \\
0.01^{* *}\end{array}$ & $\begin{array}{l}-18.63(-21.00,- \\
16.25)\end{array}$ & $\begin{array}{l}< \\
0.01^{* *}\end{array}$ \\
\hline $\mathrm{LTN}+\mathrm{HCT}$ & $\begin{array}{l}-25.79(-27.25,- \\
24.34)\end{array}$ & $\begin{array}{l}< \\
0.01^{* *}\end{array}$ & $\begin{array}{l}-25.14(-26.59 \\
-23.69)\end{array}$ & $\begin{array}{l}< \\
0.01^{* *}\end{array}$ & $\begin{array}{l}-25.42(-26.87,- \\
23.97)\end{array}$ & $\begin{array}{l}< \\
0.01^{* *}\end{array}$ & $\begin{array}{l}-26.45(-27.90,- \\
24.99)\end{array}$ & $\begin{array}{l}< \\
0.01^{* *}\end{array}$ \\
\hline N-GITS & $\begin{array}{l}-28.53(-30.07,- \\
26.989\end{array}$ & $\begin{array}{l}< \\
0.01 * *\end{array}$ & $\begin{array}{l}-28.16(-29.70,- \\
26.61)\end{array}$ & $\begin{array}{l}< \\
0.01^{* *}\end{array}$ & $\begin{array}{l}-29.58(-31.12,- \\
28.04)\end{array}$ & $\begin{array}{l}< \\
0.01^{* *}\end{array}$ & $\begin{array}{l}-28.89(-30.44,- \\
27.35)\end{array}$ & $\begin{array}{l}< \\
0.01^{* *}\end{array}$ \\
\hline $\begin{array}{l}\text { N-GITS + LTN + } \\
\text { HCT }\end{array}$ & $\begin{array}{l}-45.95(-47.92,- \\
43.97)\end{array}$ & $\begin{array}{l}< \\
0.01 * *\end{array}$ & $\begin{array}{l}-48.88(-50.85,- \\
46.9)\end{array}$ & $\begin{array}{l}< \\
0.01 * *\end{array}$ & $\begin{array}{l}-49.74(-51.72,- \\
47.77)\end{array}$ & $\begin{array}{l}< \\
0.01 * *\end{array}$ & $\begin{array}{l}-50.17(-52.15,- \\
48.20)\end{array}$ & $\begin{array}{l}< \\
0.01^{* *}\end{array}$ \\
\hline
\end{tabular}

\section{Changes in DBP from baseline}

$\begin{array}{ll}\text { LTN } & -9.25(-11.18,- \\ & 7.32) \\ \text { LTN + HCT } & -10.51(-11.69,- \\ & 9.34) \\ \text { N-GITS } & -12.32(-13.57,- \\ & 11.07) \\ \text { N-GITS + LTN }+ & -16.81(-18.41- \\ \text { HCT } & 15.21)\end{array}$

$$
\begin{array}{ll}
< & -10.00(-11.93, \\
0.01^{* *} & -8.07) \\
< & -10.33(-11.50,- \\
0.01^{* *} & 9.15) \\
< & -11.74(-12.99,- \\
0.01^{* *} & 10.49) \\
< & -16.64(-18.24,- \\
0.01^{* *} & 15.04)
\end{array}
$$$$
<\quad-9.25(-11.18,-
$$$$
0.01 * * 3.32)
$$$$
<\quad-10.00(-11.18,-
$$$$
0.01 * * 8.82)
$$

\begin{tabular}{|c|c|c|c|c|c|c|c|c|}
\hline LTN & $-9.63(-19.87,0.62)$ & 0.07 & $-5.33(-15.57,4.92)$ & 0.31 & $\begin{array}{l}-11.10(-21.35,- \\
0.85)\end{array}$ & $0.03^{*}$ & $\begin{array}{l}-13.23(-23.47,- \\
2.98)\end{array}$ & $0.01^{*}$ \\
\hline $\mathrm{LTN}+\mathrm{HCT}$ & $-4.96(-11.23,1.30)$ & 0.12 & $-7.21(-13.47,0.94)$ & $0.02^{*}$ & $\begin{array}{l}-10.73(-17.00,- \\
4.46)\end{array}$ & $\begin{array}{l}< \\
0.01^{* *}\end{array}$ & $\begin{array}{l}-13.60(-19.86,- \\
7.33)\end{array}$ & $\begin{array}{l}< \\
0.01^{* *}\end{array}$ \\
\hline N-GITS & $\begin{array}{l}-11.52(-18.17 \\
-4.87)\end{array}$ & $\begin{array}{l}< \\
0.01 * *\end{array}$ & $\begin{array}{l}-17.08(-23.73,- \\
10.43)\end{array}$ & $\begin{array}{l}< \\
0.01 * *\end{array}$ & $\begin{array}{l}-21.39(-28.04,- \\
14.74)\end{array}$ & $\begin{array}{l}< \\
0.01 * *\end{array}$ & $\begin{array}{l}-25.29(-31.95,- \\
18.64)\end{array}$ & $\begin{array}{l}< \\
0.01 * *\end{array}$ \\
\hline $\begin{array}{l}\text { N-GITS + LTN + } \\
\text { HCT }\end{array}$ & $\begin{array}{l}-13.38(-21.89 \\
-4.87)\end{array}$ & $\begin{array}{l}< \\
0.01^{* *}\end{array}$ & $\begin{array}{l}-17.62(-26.13,- \\
9.11)\end{array}$ & $\begin{array}{l}< \\
0.01^{* *}\end{array}$ & $\begin{array}{l}-14.10(-22.62,- \\
5.59)\end{array}$ & $\begin{array}{l}< \\
0.01^{* *}\end{array}$ & $\begin{array}{l}-24.93(-33.44,- \\
16.42)\end{array}$ & $\begin{array}{l}< \\
0.01 * *\end{array}$ \\
\hline \multicolumn{9}{|c|}{ Changes in Urea from baseline } \\
\hline LTN & $1.00(-0.66,2.66)$ & 0.24 & $2.35(0.69,4.01)$ & $0.01^{*}$ & $2.03(0.37,3.68)$ & $0.02^{*}$ & $2.65(0.99,4.31)$ & $\begin{array}{l}< \\
0.01^{* *}\end{array}$ \\
\hline $\mathrm{LTN}+\mathrm{HCT}$ & $0.38(-0.64,1.39)$ & 0.47 & $1.29(0.28,2.30)$ & $0.01^{*}$ & $2.19(1.17,3.20)$ & $\begin{array}{l}< \\
0.01^{* *}\end{array}$ & $3.47(2.45,4.48)$ & $\begin{array}{l}< \\
0.01 * *\end{array}$ \\
\hline N-GITS & $-0.76(-1.83,0.32)$ & 0.17 & $0.71(-0.37,1.78)$ & 0.20 & $1.39(0.31,2.47)$ & $0.01^{*}$ & $1.81(0.73,2.89)$ & $\begin{array}{l}< \\
0.01 * *\end{array}$ \\
\hline $\begin{array}{l}\text { N-GITS + LTN + } \\
\text { HCT }\end{array}$ & $0.72(-0.65,2.10)$ & 0.30 & $2.93(1.55,4.31)$ & $\begin{array}{l}< \\
0.01 * *\end{array}$ & $3.60(2.23,4.98)$ & $\begin{array}{l}< \\
0.01^{* *}\end{array}$ & $5.16(3.78,6.53)$ & $\begin{array}{l}< \\
0.01^{* *}\end{array}$ \\
\hline \multicolumn{9}{|c|}{ Changes in Creatinine from baseline } \\
\hline LTN & $0.02(-0.03,0.06)$ & 0.45 & $0.03(-0.02,0.07)$ & 0.28 & $0.05(-0.001,0.09)$ & 0.06 & $0.07(0.02,0.11)$ & $\begin{array}{l}< \\
0.01^{* *}\end{array}$ \\
\hline $\mathrm{LTN}+\mathrm{HCT}$ & $0.01(-0.02,0.03)$ & 0.74 & $0.02(-0.01,0.05)$ & 0.11 & $0.02(-0.01,0.05)$ & 0.11 & $0.04(0.01,0.07)$ & $\begin{array}{l}< \\
0.01 * *\end{array}$ \\
\hline N-GITS & $-0.03(-0.06,0.003)$ & 0.08 & $-0.02(-0.05,0.01)$ & 0.11 & $-0.04(-0.07,-0.01)$ & $0.01^{*}$ & $-0.04(-0.07,-0.01)$ & $0.01^{*}$ \\
\hline $\begin{array}{l}\text { N-GITS + LTN + } \\
\text { HCT }\end{array}$ & $-0.01(-0.05,0.02)$ & 0.47 & $0.01(-0.03,0.04)$ & 0.72 & $0.04(0.002,0.08)$ & $0.04^{*}$ & $0.07(0.03,0.10)$ & $\begin{array}{l}< \\
0.01^{* *}\end{array}$ \\
\hline
\end{tabular}$$
<\quad-11.58(-12.83,-
$$$$
\left.0.01^{* *} \quad 10.33\right)
$$

$<\quad-16.90(-18.50,-$

$\left.0.01^{* *} \quad 15.30\right)$

$\begin{array}{lll}< & -9.75(-11.68,- & < \\ 0.01^{* *} & 7.82) & 0.01^{* *} \\ < & -10.89(-12.07,- & < \\ 0.01^{* *} & 9.71) & 0.01^{* *} \\ < & -12.21(-13.46,- & < \\ 0.01^{* *} & 10.96) & 0.01^{* *} \\ < & -16.55(-18.15,- & < \\ 0.01^{* *} & 14.95) & 0.01^{* *}\end{array}$

\section{Changes in BSR from baseline}

Abbreviations: LTN losartan potassium, N-GITS Nifedipine GITS, HCT hydrochlorothiazide, SBP systolic blood pressure, DBP diastolic blood pressure, BSR blood sedimentation rate

p-values: ${ }^{*} \leq 0.05,{ }^{* *}<0.01$

percentage of women with hypertension is higher compared to men [23]. We also observed that $60 \%$ hypertensive women were between 50 and 64 years of age compared to only $20.3 \%$ hypertensive women under the age of 50 years. Several previous researches have been conducted to assess dietary approaches in the 
Table 4 Mean Changes in Outcomes Measures; Low vs High Risk Women, Adjusted for Treatment Effects

\begin{tabular}{|c|c|c|c|c|c|c|c|c|}
\hline Changes & 3 Months & & 6 Months & & 9 Months & & 12 Months & \\
\hline $\begin{array}{l}\text { in } \\
\text { Outcome } \\
\text { Measures } \\
\text { from } \\
\text { Baseline; } \\
\text { Low vs. } \\
\text { High Risk }\end{array}$ & $\beta(95 \% \mathrm{Cl})$ & $p$-value & $\beta(95 \% \mathrm{Cl})$ & $\boldsymbol{p}$-value & $\beta(95 \% \mathrm{Cl})$ & $\boldsymbol{p}$-value & $\beta(95 \% \mathrm{Cl})$ & $\boldsymbol{p}$-value \\
\hline
\end{tabular}

\section{Changes in SBP relative to baseline}

\begin{tabular}{|c|c|c|c|c|c|c|c|c|}
\hline Low risk & $\begin{array}{l}-17.87(-20.53,- \\
15.23)\end{array}$ & $\begin{array}{l}< \\
0.01^{* *}\end{array}$ & $\begin{array}{l}-17.87(-20.51,- \\
15.24)\end{array}$ & $\begin{array}{l}< \\
0.01^{* *}\end{array}$ & $\begin{array}{l}-16.53(-19.17,- \\
13.88)\end{array}$ & $\begin{array}{l}< \\
0.01 * *\end{array}$ & $\begin{array}{l}-17.92(-20.56,- \\
15.28)\end{array}$ & $\begin{array}{l}< \\
0.01 * *\end{array}$ \\
\hline $\begin{array}{l}\text { High } \\
\text { risk }\end{array}$ & $\begin{array}{l}-17.87(-20.34,- \\
15.40)\end{array}$ & $\begin{array}{l}< \\
0.01^{* *}\end{array}$ & $\begin{array}{l}-17.87(-20.38,- \\
15.36)\end{array}$ & $\begin{array}{l}< \\
0.01^{* *}\end{array}$ & $\begin{array}{l}-16.19(-18.69,- \\
13.68)\end{array}$ & $\begin{array}{l}< \\
0.01 * *\end{array}$ & $\begin{array}{l}-19.48(-21.99,- \\
16.97)\end{array}$ & $\begin{array}{l}< \\
0.01 * *\end{array}$ \\
\hline \multicolumn{9}{|c|}{ Change between (ref: Low risk) } \\
\hline $\begin{array}{l}\text { High } \\
\text { risk }\end{array}$ & $0.006(-1.32,1.33)$ & 0.98 & $0.009(-1.29,1.30)$ & 0.99 & $0.34(-1.01,1.69)$ & 0.62 & $-1.55(-2.92,-0.18)$ & $0.03^{*}$ \\
\hline
\end{tabular}

Changes in DBP relative to baseline

$\begin{array}{lllllllll}\text { Low risk }-8.49(-10.63,-6.35) & < & -10.17(-12.28,- & < & -9.25(-11.38,-7.13) & < & -9.49(-11.62,-7.37) & < \\ & & 0.01^{* *} & 8.05) & 0.01^{* *} & & 0.01^{* *} & 0.01^{* *} \\ \text { High }-9.97(-11.97,-7.98) & < & -9.84(-11.87,-7.82) & < & -9.31(-11.33,-7.28) & < & -10.12(-12.15,- & < \\ \text { risk } & 0.01^{* *} & & 0.01^{* *} & & 0.01^{* *} & 8.10) & 0.01^{* *}\end{array}$

Change between (ref: Low risk)

\begin{tabular}{|c|c|c|c|c|}
\hline $\begin{array}{l}\text { High } \\
\text { risk }\end{array}$ & $-1.57(-2.60,-0.54)$ & $\begin{array}{l}< \\
0.01 * *\end{array}$ & $0.24(-0.78,1.25)$ & 0.65 \\
\hline
\end{tabular}

Changes in BSR relative to baseline

\begin{tabular}{lllllllll} 
Low risk & $-7.13(-18.65,4.40)$ & 0.23 & $-1.81(-13.31,9.68)$ & 0.76 & $-8.75(-20.25,2.76)$ & 0.14 & $-11.17(-22.67,0.34)$ & 0.06 \\
$\begin{array}{l}\text { High } \\
\text { risk }\end{array}$ & $-10.16(-20.86,0.53)$ & 0.06 & $-7.00(-17.91,3.90)$ & 0.21 & $-11.30(-22.17,-$ & $0.04^{*}$ & $-13.06(-23.95,-2.17)$ & $0.02^{*}$ \\
\hline
\end{tabular}

Change between (ref: Low risk)

$\begin{array}{llllllll}\text { High } & 0.98(-4.99,6.95) & 0.75 & -1.17(-7.07,4.73) & 0.70 & 1.46(-4.67,7.59) & 0.64 & 2.12(-4.09,8.34)\end{array}$

risk

\begin{tabular}{|c|c|c|c|c|c|c|c|c|}
\hline Low risk & $1.06(-0.81,2.93)$ & 0.27 & $2.49(0.62,4.35)$ & $0.01^{*}$ & $2.04(0.18,3.91)$ & $0.03^{*}$ & $2.83(0.97,4.70)$ & $<0.01^{* *}$ \\
\hline $\begin{array}{l}\text { High } \\
\text { risk }\end{array}$ & $1.00(-0.73,2.73)$ & 0.26 & $2.27(0.50,4.03)$ & $0.01^{*}$ & $2.09(0.33,3.85)$ & $0.02^{*}$ & $2.51(0.75,4.28)$ & $0.01^{*}$ \\
\hline \multicolumn{9}{|c|}{ Change between (ref: Low risk) } \\
\hline $\begin{array}{l}\text { High } \\
\text { risk }\end{array}$ & $0.07(-0.90,1.04)$ & 0.89 & $-0.09(-1.05,0.87)$ & 0.86 & $0.18(-0.82,1.17)$ & 0.73 & $-0.19(-1.20,0.82)$ & 0.71 \\
\hline \multicolumn{9}{|c|}{ Changes in Creatinine relative to baseline } \\
\hline Low risk & $0.01(-0.04,0.06)$ & 0.77 & $0.01(-0.04,0.07)$ & 0.58 & $0.05(0.004,0.11)$ & $0.04^{*}$ & $0.07(0.02,0.12)$ & $0.01^{*}$ \\
\hline $\begin{array}{l}\text { High } \\
\text { risk }\end{array}$ & $0.01(-0.02,0.03)$ & 0.57 & $0.03(-0.01,0.08)$ & 0.17 & $0.03(-0.02,0.08)$ & 0.26 & $0.06(0.01,0.11)$ & $0.01^{*}$ \\
\hline \multicolumn{9}{|c|}{ Change between (ref: Low risk) } \\
\hline $\begin{array}{l}\text { High } \\
\text { risk }\end{array}$ & $0.008(-0.02,0.03)$ & 0.57 & $0.01(-0.01,0.04)$ & 0.40 & $-0.03(-0.06,-0.01)$ & $0.01^{*}$ & $-0.01(-0.04,0.01)$ & 0.36 \\
\hline
\end{tabular}

Abbreviations: LTN losartan potassium, N-GITS Nifedipine GITS, HCT hydrochlorothiazide, SBP systolic blood pressure, DBP diastolic blood pressure, BSR blood sedimentation rate

$p$-values ${ }^{*} \leq 0.05,{ }^{* *}<0.01$

management of hypertension and to estimate an association between diet and hypertension [24], yet the association cannot be ascribed to a single food item or nutrient which makes it a more composite risk factor in South Asians due to considerable variations in diet within and between South Asian population [25]. In this context, salt consumption in South Asians are generally higher that not only effects blood pressure but also increases the risk of stroke and cardiovascular diseases by altering arterial stiffness - contributing towards resistant 
hypertension in patients that are considered salt sensitive [26]. We found that compared to low salt consumption, patients consuming normal salt were considered high risk population corroborating findings of a systematic review that consuming lower salt can reduce blood pressure and subsequent risk of cardiovascular disease [27]. Fruits and vegetables intake have been shown to reduce blood pressure in number of studies [28], however, a study from Pakistan showed no association between fruit and vegetables consumption and lower risk of hypertension [24]. We found that compared to low intake, higher consumption of vegetables and fruits, red/ white meat and high fat diet could frame women as high risk population. This could possibly be ascribed to the cooking methods used by South Asians, i-e., stir frying, overcooking of vegetables, use of animal saturated fats/ desi ghee (extracted from butter) in daily vegetables cooking and poor ascertainment regarding consumption of fruits and vegetables, whether cooked or uncooked. Our data regarding meat consumption and risk of hypertension is in complete agreement with previously reported data that higher consumption of meat, particularly red, is strongly associated with higher risk of cardiovascular diseases in women [29].

According to JNC 8 guidelines, first line antihypertensive therapy should consist of thiazide-type diuretic, calcium channel blockers, angiotensin-converting enzyme inhibitor (ACEIs), or an angiotensin receptor blocker (ARB) [30]. The European society of hypertension and European society of cardiology guidelines recommend the use of combination therapy in majority of patients with calcium channel blockers being the most preferred combination [11]. Likewise, randomized and observational studies have shown that CCB, Nifedipine-GITS, is effective both as monotherapy and in combination [31, 32]. In this context, the TALENT study demonstrated that the use of Nifedipine GITS $20 \mathrm{mg}$ in combination with telmisartan $80 \mathrm{mg}$ provided better and earlier blood pressure control compared to monotherapy [31]. Similarly, AdADOSE, a 12 week multicenter, prospective, observational study, suggested that a combination therapy with Nifedipine-GITS was more effective in reducing systolic (SBP) and diastolic blood pressure (DBP) compared to other therapeutic choices along with low frequency of treatment related adverse effects [17]. Similar to these findings, we found that Nifedipine-GITS, compared to baseline, either in combination or alone, demonstrated significant reductions in SBP and DBP starting from 1st follow till the last follow up, yet maximum reduction was observed when Nifedipine-GITS was used in combination with other drugs, i-e., losartan (LTN) and hydrochlorothiazide (HCT). Moreover, changes in blood sedimentation rate (BSR) with Nifedipine-GITS alone or in combination was almost similar. Compared to combination therapy, Nifedipine-GITS alone exhibited minimal changes in urea and creatinine levels as reported previously [33]. Additionally, Nefidipine-GITS alone exhibited minimal side effects, such as dry cough, headache, orthostatic hypotension and odema, however when used with fixed dose combination, losartan and hydrochlorothiazide, orthostatic hypotension was significantly higher followed by odema. Nonetheless, contrary to our findings, combination of Nifedipine-GITS and candesartan exhibited improved safety profiles with lower incidence of vasodilatory adverse effects, such as odema, and headache [34]. In another study, ADVISE, combination of Nifedipine GITS + Valsartan compared to Valsartan alone demonstrated better and consistent blood pressure control in Asian population [15], but with a few vasodilatory side effects, as observed in our study, mostly with combination therapy, i-e., N-GITS + LTN + HCT. Hence, the more sever vasodilatory adverse events in our study, odema and orthostatic hypotension, could be attributed to the use of fixed dose triple combination, i-e., losartan, hydrochlorothiazide in combination with Nifedipine GITS. Thus, data from our study and of others clearly demonstrated that Nifedipine GITS in combination with angiotensin II receptor blockers and hydrochlorothiazide rendered better blood pressure control in hypertensive patients, particularly women, but may increase the frequency of vasodialtory effects.

\section{Study limitations}

Our study has a few limitations; observational design, subjects were observed over a period of time but were not allowed to intervene, and collection of data from a single tertiary care facility that may limit the generalization of study results. Additionally, no information was available regarding financial and other stressors affecting blood pressure control. Similarly, information on the use of traditional remedies, very common in Pakistan, and their contribution in the control of blood pressure cannot be ascertained. Moreover, not a single literature report from Pakistan on the studied topic was available to directly compare our results.

\section{Implications for practice/policy}

Pakistan, a male dominant society, where females seldom enjoy full rights and access to opportunities with regards to very basic needs. The situation is even worse in health sector due to lack of female doctors and cultural forces limiting the access to health facilities. In Pakistan, hypertensive males and females, irrespective of risk level, are treated using routine but similar treatment algorithms and non-pharmacological approaches. Additionally, not a single primary or tertiary care facility utilizes gender specific anti-hypertensive protocols. Our findings, the first report from Pakistan, clearly 
demonstrated that in high risk hypertensive women, compared to losartan, Nifedipine-GITS would be more suitable single agent choice with minimal side effects. Moreover, combining Nifedipine-GITS with fixed dose combination, Losartan + Hydrochlorothiazide, would provide improved blood pressure control in sever and resistant/high risk hypertensive women. Thus, these findings would be of interest to clinicians in implementing lifestyle modifications and selection of antihypertensive therapy in hypertensive women of Pakistan, and would also impact anti-hypertensive drug enlistment and procurement criterion, particularly for hospitals with higher influx of hypertensive women.

\section{Conclusion}

In conclusion, our data clearly demonstrated that nifedipine-GITS alone is more efficacious in lowering systolic and diastolic BP in high and low-risk female patients than losartan alone, with improved renal protection. Additionally, the combination of nifedipine GITS with fixed dose losartan + HCT had improved blood pressure lowering effects but with higher frequency of vasodilatory side effects, such as orthostatic hypotension and edema. Thus, it may be appropriate to initially manage high risk hypertensive women on a single agent, i-e., Nifedipine-GITS in comparison to Losartan, having better clinical efficacy and safety profiles, if counselled properly with regards to life style and dietary modifications.

\section{Supplementary information}

Supplementary information accompanies this paper at https://doi.org/10. 1186/s12905-020-01033-2.

\section{Additional file 1 Table S1. Population clinical and laboratory}

biochemical values of four anti-hypertensive therapies; baseline vs follow ups. Figure S1. Macrograph Showing Frequency of Co-morbidities in Hypertensive Women of Lahore, Punjab, Pakistan. Figure S2. Macrograph Showing Frequency of Therapy Related Adverse Effects in Hypertensive Women.

\section{Abbreviations}

Nifedipine-GITS: Nifedipine gastrointestinal system; HIS: Hospital information system; SBP: Systolic blood pressure; ACEls: Angiotensin converting enzyme inhibitor; BP: Blood pressue; LTN: Losartan potassium;

HCT: Hydrochlorothiazide; DBP: Diastolic blood pressure; ADR: Adverse drug reaction; GLM: Generalized linear regression model; SPSS: Statistical software for the social sciences; BSR: Blood sedimentation rate; CVDs: Cardiovascular diseases; CCB: Calcium channel blocker; ARB: Angiotensin receptor blockers; OR: Odds ratio; JNC6: Joint national committee 6
\end{abstract}

\section{Authors' contributions}

MU; collected the data and analyzed data, MA; designed the study and provided the logistics, HS; designed the study, analyzed data and wrote the manuscript, ZS; designed the study and edited the manuscript, FT; analyzed the data and edited the manuscript. All the authors have read and approved the manuscript.

\section{Funding}

This study received no finds from any institution or department, governmental or private.

\section{Availability of data and materials}

The data sets used in the study can be provided upon request to corresponding author

\section{Ethics approval and consent to participate}

Ethical approval for the study was obtained from Ethical Committee on human research, University College of Pharmacy, University of the Punjab, reference \#, ECCR/UCP/067/2016, and Hospital committee of ethics on human research. Informed written consent was obtained from all the enrollees by a collaborating medical doctor of the hospital.

\section{Consent for publication \\ Not Applicable.}

\section{Competing interests}

Authors declared that they have no conflicts of interest.

\section{Author details}

${ }^{1}$ Section of Pharmacology, University College of Pharmacy, University of the Punjab, Allama lqbal Campus, Lahore 54000, Pakistan. ${ }^{2}$ Section of

Pharmaceutics, University of the Punjab, Allama lqbal Campus, Lahore 54000, Pakistan. ${ }^{3}$ University of Lahore, Lahore, Pakistan. ${ }^{4}$ Institute of Health and Society, Department of Medicine, University of Oslo, Oslo, Norway.

Received: 21 October 2019 Accepted: 26 July 2020

Published online: 01 August 2020

\section{References}

1. Organization WH. Global Health Observatory (GHO) data 2015. Retrieved. 2015;3:23.

2. Lim SS, Vos T, Flaxman AD, Danaei G, Shibuya K, Adair-Rohani H, Amann M, Anderson HR, Andrews KG, Aryee M, et al. A comparative risk assessment of burden of disease and injury attributable to 67 risk factors and risk factor clusters in 21 regions, 1990-2010: a systematic analysis for the global burden of disease study 2010. Lancet. 2012;380(9859):2224-60.

3. Smulyan H, Asmar RG, Rudnicki A, London GM, Safar ME. Comparative effects of aging in men and women on the properties of the arterial tree. J Am Coll Cardiol. 2001;37(5):1374-80.

4. Ljungman C, Kahan T, Schioler L, Hjerpe P, Hasselstrom J, Wettermark B, Bostrom KB, Manhem K. Gender differences in antihypertensive drug treatment: results from the Swedish primary care cardiovascular database (SPCCD). J Am Soc Hypertens. 2014;8(12):882-90.

5. Keyhani S, Scobie JV, Hebert PL, McLaughlin MA. Gender disparities in blood pressure control and cardiovascular care in a national sample of ambulatory care visits. Hypertension. 2008;51(4):1149-55.

6. Gu Q, Burt VL, Paulose-Ram R, Dillon CF. Gender differences in hypertension treatment, drug utilization patterns, and blood pressure control among US adults with hypertension: data from the National Health and nutrition examination survey 1999-2004. Am J Hypertens. 2008;21(7):789-98.

7. Daugherty SL, Masoudi FA, Ellis JL, Ho PM, Schmittdiel JA, Tavel HM, Selby JV, O'Connor PJ, Margolis KL, Magid DJ. Age-dependent gender differences in hypertension management. J Hypertens. 2011;29(5):1005-11.

8. Levy D, Larson MG, Vasan RS, Kannel WB, Ho KK. The progression from hypertension to congestive heart failure. Jama. 1996;275(20):1557-62.

9. Llisterri JL, Barrios V de la Sierra A, Bertomeu V. Escobar C, Gonzalez-Segura D. Blood pressure control in hypertensive women aged 65 years or older in a primary care setting. MERICAP study. Rev Esp Cardiol. 2011;64(8):654-60.

10. Turnbull F, Woodward M, Neal B, Barzi F, Ninomiya T, Chalmers J, Perkovic V, Li N, MacMahon S. Do men and women respond differently to blood pressure-lowering treatment? Results of prospectively designed overviews of randomized trials. Eur Heart J. 2008;29(21):2669-80.

11. Mancia G, De Backer G, Dominiczak A, Cifkova R, Fagard R, Germano G, Grassi G, Heagerty AM, Kjeldsen SE, Laurent S, et al. 2007 guidelines for the Management of Arterial Hypertension: the task force for the Management of Arterial Hypertension of the European Society of Hypertension (ESH) and of the European Society of Cardiology (ESC). J Hypertens. 2007;25(6):1105-87. 
12. Lundy A, Lutfi N, Beckey C. Review of nifedipine GITS in the treatment of high risk patients with coronary artery disease and hypertension. Vasc Health Risk Manag. 2009;5(1):429-40.

13. Brown MJ, Palmer CR, Castaigne A, de Leeuw PW, Mancia G, Rosenthal T, Ruilope LM. Morbidity and mortality in patients randomised to double-blind treatment with a long-acting calcium-channel blocker or diuretic in the international Nifedipine GITS study: intervention as a goal in hypertension treatment (INSIGHT). Lancet. 2000;356(9227):366-72.

14. Poole-Wilson PA, Lubsen J, Kirwan BA, van Dalen FJ, Wagener G, Danchin N, Just H, Fox KA, Pocock SJ, Clayton TC, et al. Effect of long-acting nifedipine on mortality and cardiovascular morbidity in patients with stable angina requiring treatment (ACTION trial): randomised controlled trial. Lancet. 2004; 364(9437):849-57

15. Ke YN, Dong YG, Ma SP, Yuan H, Ihm SH, Baek SH. Improved blood pressure control with Nifedipine GITS/valsartan combination versus high-dose valsartan Monotherapy in mild-to-moderate hypertensive patients from Asia: results from the ADVISE study, a randomized trial. Cardiovasc Ther. 2012;30(6):326-32.

16. Pimenta E. Hypertension in women. Hypertens Res. 2012;35(2):148-52.

17. Motaweih AK, Usova E, Hussain W, Dello Z, Schmidt B, Petri T. Effectiveness of combination therapy with nifedipine GITS: a prospective, 12-week observational study (AdADOSE). BMC Cardiovasc Disord. 2015;15:35.

18. James PA, Oparil S, Carter BL, Cushman WC, Dennison-Himmelfarb C, Handler J, Lackland DT, LeFevre ML, MacKenzie TD, Ogedegbe O. 2014 evidence-based guideline for the management of high blood pressure in adults: report from the panel members appointed to the eighth joint National Committee (JNC 8). Jama. 2014;311(5):507-20.

19. Kish L. Survey sampling; 1965

20. Shafi ST, Shafi T. A survey of hypertension prevalence, awareness, treatment, and control in health screening camps of rural Central Punjab, Pakistan. J Epidemiol Glob Health. 2017;7(2):135-40.

21. Fauji Foundation Hospital Lahore [https://www.lcci.pk/listing/faujifoundation-hospital-lahore/]. Accessed 4 Sept 2019.

22. Reddy KS, Katan MB. Diet, nutrition and the prevention of hypertension and cardiovascular diseases. Public Health Nutr. 2004;7(1a):167-86.

23. Roger VL, Go AS, Lloyd-Jones DM, Adams RJ, Berry JD, Brown TM, Carnethon MR, Dai S, de Simone G, Ford ES, et al. Heart disease and stroke statistics--2011 update: a report from the American Heart Association. Circulation. 2011;123(4):e18-e209.

24. Safdar NF, Bertone-Johnson ER, Cordeiro L, Jafar TH, Cohen NL. Dietary patterns and their association with hypertension among Pakistani urban adults. Asia Pac J Clin Nutr. 2015;24(4):710-9.

25. Gupta M, Brister S, Verma S. Is south Asian ethnicity an independent cardiovascular risk factor? Can J Cardiol. 2006;22(3):193-7.

26. Park JB, Kario K, Wang JG. Systolic hypertension: an increasing clinical challenge in Asia. Hypertens Res. 2015;38(4):227-36.

27. He FJ, MacGregor GA. Effect of longer-term modest salt reduction on blood pressure. Cochrane Database Syst Rev. 2004;3(3):1-49.

28. Ascherio A, Hennekens C, Willett WC, Sacks F, Rosner B, Manson J, Witteman J, Stampfer MJ. Prospective study of nutritional factors, blood pressure, and hypertension among US women. Hypertension. 1996;27(5):1065-72.

29. Bovalino S, Charleson G, Szoeke C. The impact of red and processed meat consumption on cardiovascular disease risk in women. Nutrition. 2016;32(3): 349-54.

30. James PA, Oparil S, Carter BL, Cushman WC, Dennison-Himmelfarb C, Handler J, Lackland DT, LeFevre ML, MacKenzie TD, Ogedegbe O, et al. 2014. 2014 Evidence-Based Guideline for the Management of High Blood Pressure in Adults. JAMA. 2014;311(5):507-520. https://doi.org/10.1001/jama. 2013.284427.

31. Mancia G, Parati G, Bilo G, Choi J, Kilama MO, Ruilope LM. Blood pressure control by the nifedipine GITS-telmisartan combination in patients at high cardiovascular risk: the TALENT study. J Hypertens. 2011;29(3):600-9.

32. Hasebe N, Kikuchi K. Controlled-release nifedipine and candesartan lowdose combination therapy in patients with essential hypertension: the NICE combi (Nifedipine and candesartan combination) study. J Hypertens. 2005; 23(2):445-53.

33. Kita T, Yokota N, Ichiki Y, Ayabe T, Etoh T, Tamaki N, Kato J, Eto T, Kitamura K. One-year effectiveness and safety of open-label losartan/ hydrochlorothiazide combination therapy in Japanese patients with hypertension uncontrolled with ARBs or ACE inhibitors. Hypertens Res. 2010; 33(4):320-5.
34. Kjeldsen SE, Sica D, Haller H, Cha G, Gil-Extremera B, Harvey P, Heyvaert F, Lewin AJ, Villa G, Mancia G. Nifedipine plus candesartan combination increases blood pressure control regardless of race and improves the side effect profile: DISTINCT randomized trial results. J Hypertens. 2014;32(12): 2488-98 discussion 2498.

\section{Publisher's Note}

Springer Nature remains neutral with regard to jurisdictional claims in published maps and institutional affiliations.
Ready to submit your research? Choose BMC and benefit from:

- fast, convenient online submission

- thorough peer review by experienced researchers in your field

- rapid publication on acceptance

- support for research data, including large and complex data types

- gold Open Access which fosters wider collaboration and increased citations

- maximum visibility for your research: over $100 \mathrm{M}$ website views per year

At BMC, research is always in progress.

Learn more biomedcentral.com/submissions 